\title{
Associations of Healthcare Utilization and Costs with Increasing Pain and Treatment Intensity Levels in Osteoarthritis Patients: an 18-Year Retrospective Study
}

Jove Graham ( $\square$ jhgraham1@geisinger.edu )

Geisinger Health System

Tonia Novosat

Geisinger Health System

Haiyan Sun

Geisinger Health System

Brian J. Piper

Geisinger Health System

Joseph A. Boscarino

Geisinger Health System

Melissa Kern

Geisinger Health System

Vanessa Hayduk

Geisinger Health System

Eric Wright

Geisinger Health System

Craig Beck

Pfizer (United States)

Rebecca Robinson

Eli Lilly (United States)

Edward Casey

Pfizer (United States)

Jerry Hall

Eli Lilly (United States)

\section{Patricia Schepman}

Pfizer (United States)

\section{Research Article}

Keywords: osteoarthritis, pain, health economics, clinical phenotyping

Posted Date: December 28th, 2021

DOl: https://doi.org/10.21203/rs.3.rs-1196794/v1 
License: (a) (1) This work is licensed under a Creative Commons Attribution 4.0 International License. Read Full License 


\section{Abstract}

\section{Background}

Osteoarthritis $(\mathrm{OA})$ is a complex disease, and prior studies have documented the health and economic burdens of patients with OA compared to those without OA. Our goal was to use two strategies to further stratify OA patients based on both pain and treatment intensity to examine healthcare utilization and costs using electronic records from 2001-2018 at a large integrated health system.

\section{Methods}

Adult patients with $\geq 1$ pain numerical rating score (NRS) and diagnosis of OA were included. Pain episodes of $\geq 90$ days were defined as mild (0-3), moderate (4-6) or severe (7-10) based on initial NRS. Patients were initially classified as mild and moved to moderate-severe OA if any of eight treatment-based criteria were met. Outpatient visits (OP), emergency department visits (ED), inpatient days, and healthcare costs (both all-cause and OA-specific) were compared among pain levels and OA severity levels as frequencies and per-member-per-year rates, using generalized linear regression models adjusting for age, sex and body mass index, with contrasts of $p<0.05$ considered significant.

\section{Results}

We identified 127,656 patients, 92,576 with pain scores. Moderate and severe pain were associated with significantly higher rates of OA-related utilization and costs, and all-cause ED visits and pharmacy costs. Moderate-severe OA patients had significantly higher OA-related utilization and costs, and all-cause OP, ED and pharmacy costs.

\section{Conclusions}

Pain and treatment intensity were both strongly associated with OA-related resource utilization but not consistently with all-cause utilization. With better understanding of how OA patients intensify services, thus increasing costs, we can deploy targeted preventative strategies aimed at halting progression into more costly phases of the disease.

\section{Introduction}

Osteoarthritis (OA) is a mechanical and inflammatory mediated disease that involves progressive change in the external and internal environment of the joint and results in substantial clinical and economic burden. ${ }^{1,2}$ Worldwide, $14 \%$ of all individuals over age 60 have this diagnosis, ${ }^{3}$ and prevalence is only anticipated to increase. ${ }^{4}$ In 2030 , it is projected that approximately $25 \%$ of American adults will have disability due to OA, and over $50 \%$ of these sufferers will be 65 years of age and older. ${ }^{4}$ In the United States (U.S.), hospitalizations for arthritis are the second most expensive, costing roughly $\$ 20$ billion, ${ }^{5}$ and the U.S. is estimated to spend $\$ 139.8$ billion annually on outpatient OA care $^{6-8}$ In addition to age, there are also clear associations between OA and risk factors such as obesity, and female $\operatorname{sex}^{9-15}$

The most likely reason for patients with osteoarthritis to seek help from a physician is the symptom of pain. ${ }^{16}$ However, due to inconsistent measures to qualify pain sensation, as well as the perception that joint pain is a normal consequence of aging, many patients suffer in silence. ${ }^{17}$ Even though self-reported pain scores such as the Numerical Rating Scale (NRS) are limited in adequately describing the multidimensional nature of pain, a two point difference (on a 0-10 scale) has been proven to detect clinically important changes in pharmaceutical and physical 
therapy trials, ${ }^{18,19}$ and using the electronic health records to consistently report this measure has been shown to improve pain management outcomes. ${ }^{20}$ Recently, increased healthcare resource utilization in patients with knee and hip OA was shown to be associated with higher pain scores, with cost of care for patients with mild pain roughly $18 \%$ less than for those who rated their pain as moderate to severe. ${ }^{21}$

Osteoarthritis pain is not always correlated with disease progression, ${ }^{16,22}$ however, as the pathogenesis of OA is labile. This lability makes it difficult to reliably determine disease severity, particularly when imaging studies are clinically not indicated to make an OA diagnosis and, thus, not routinely ordered. ${ }^{23}$ Although significant strides have been made in evidence-based clinical algorithms and care guidelines have been established, the data to guide clinical treatment decision making is still limited. ${ }^{8,24-27}$

Understanding the burden of $\mathrm{OA}$ is key to determining the most beneficial preventative strategies and the potential therapeutic interventions that would have the most beneficial impact. ${ }^{28,29}$ Our objective was to utilize two strategies for segmenting patients into OA severity groups-one defined by pain scores, and one based on treatment intensityand compare how these two strategies were able to differentiate between groups having different healthcare utilization and costs. This is the first study to our knowledge to use both electronic health records and claims to analyze direct financial costs in subcategories of disease severity, based on both pain and treatment progression, in a large open-cohort design in an integrated health system over an 18-year period.

\section{Methods}

\section{Setting and Study Design}

All data for this study originated from Geisinger, an integrated health system in Pennsylvania. Geisinger serves over 500,000 patients per year with seven hospitals, a network of 138 primary and specialty clinics, and a single electronic health record (EHR) platform (Epic Corporation, Verona, WI) encompassing inpatient and outpatient care across its network since 2001. Geisinger Health Plan, an affiliated insurance company, provides insurance to approximately one-third of the patients receiving care at Geisinger, with the remaining patients having a mix of commercial, government, and other insurance plans.

This was a retrospective, open-cohort study using EHR and insurance claims at Geisinger. The aim was to compare how two strategies for segmenting OA patients based on pain or treatment intensity were able to differentiate between groups having different healthcare utilization and costs. Patients were initially identified as eligible for the study if they had at least two outpatient encounters at a Geisinger facility between January 1, 2001 and December 31,2018 and were age 18 or older when they received a diagnosis code for OA of any joint in the EHR on an encounter, problem list, or OA-related procedure (hip/knee replacement, arthroscopy or injection). The first occurrence of an OA diagnosis or procedure was defined as the patient's index date, recognizing that in a retrospective study like this one, patients may have been diagnosed with OA prior to the study period or prior to entering the health system. OA was defined using the International Classification of Diseases Ninth/Tenth Revisions, Clinical Modification (ICD-9/10-CM) codes ICD-9: 715.* or ICD-10 M15-19. ${ }^{30,31}$ Other procedure codes are listed in supplementary Table X1 in the Appendix. For this study, patients were included in the treatment intensitybased analysis if they had $\geq 1$ month of insurance coverage and were included in the pain analysis if they also had $\geq 1$ NRS for pain after OA diagnosis. Patients were right-censored when they dropped insurance enrollment, died, or reached the end of the study period on December 31, 2018, whichever came first. The study was reviewed and approved by Geisinger's Institutional Review Board (IRB). 


\section{Pain Episode Definition}

We examined NRS pain scores (scale of 0-10) taken on or after the first OA diagnosis in either the inpatient or outpatient setting, and defined "pain episodes" for every patient, where patients could contribute multiple episodes to the analysis. Each pain episode began on the date when an NRS score was taken, and continued for 90 days, with time-dependent covariates such as age or body mass index (BMI) updated accordingly. If an additional NRS was taken during that 90 days, the length of the episode was extended further until 90 days elapsed with no new NRS scores. In order to avoid double counting utilization or cost, episodes could not overlap, and each episode was categorized based on the initial pain score taken as mild (0-3), moderate (4-6) or severe (7-10). These categories have been widely used in clinical studies and routine clinical practice. ${ }^{32}$

\section{Treatment Stage Definition}

We defined two stages of OA progression based on treatment intensity, mild and moderate-severe. A set of eight treatment-based criteria identified in EHR and claims data were used to define moderate-severe $O A$, and these were based on the 2019 OA treatment recommendations from the American College of Rheumatology (ACR) and 2019 OA treatment algorithm from the European Society for Clinical and Economic Aspects of Osteoporosis and Osteoarthritis (ESCEO) and other literature. ${ }^{24-26,33-39}$ These criteria are outlined and described in Table 1. At the time of initial OA diagnosis (index date), each patient was initially assigned to the mild category. If a patient never met any of the criteria, their entire timespan was classified as mild $O A$, and if a patient met at least one of the criteria on the same date as their first OA diagnosis, then their entire timespan was classified as moderate-severe OA.

Otherwise, if a criterion was met after initial diagnosis, the patient's time period was divided into mild and moderatesevere periods (i.e., the patient was presumed to progress to moderate-severe $\mathrm{OA}$ after the criterion was met). As before, time-dependent covariates such as age or BMI were updated at the start of each period. Kaplan-Meier survival analysis was used to examine and plot the percentage of patients progressing to moderate-severe OA over time.

As a secondary analysis to empirically support the validity of the eight criteria, we compared the prevalence of each criterion between two subgroups of patients: (1) "incident TKR patients," with total knee replacement (TKR) and at least 24 months of EHR records prior to first OA diagnosis; and (2) "no THR" patients who received no hip or knee replacement. Any patients not meeting either definition were excluded from this specific analysis. Each patient was flagged as to whether they met each of the eight criteria as of 180 days prior to TKR (group 1) or last encounter in the EHR (group 2). We hypothesized that incident TKR patients would be significantly more likely than the comparison group to have experienced each criterion if it were a marker for moderate-severe OA. A logistic regression model was used to estimate the relative risk ratios (RRR) and $95 \%$ confidence intervals (Cl) of those ratios between the two groups.

\section{Statistical Analysis}

Descriptive statistics (means and percentages) with standardized differences were used to describe the baseline characteristics of our two main populations (insured patients for the treatment-based analysis, and insured patients with pain episodes for the pain-based analysis), as well as the original EHR-based cohort these were drawn from. We compared age, sex, body mass index (BMI) and Charlson Comorbidity Index scores (CCl) between treatmentbased and pain episode groups. Once the start and end dates of pain episodes and treatment severity stages were established, we compared patients in the different categories with respect to all-cause outpatient visits (OP), emergency department visits (ED), and inpatient days hospitalized (IP). For these utilization outcomes, we analyzed 
both the frequency of use (percentage of time periods in which any use occurred) and the units used, expressed as rates per member per year (PMPY). For costs, we tabulated the total allowed amounts (i.e., amounts actually paid to the provider, combining payor and patient deductible, insurance and out-of-pocket costs) in the following categories: total cost, pharmacy cost, and medical cost, with medical cost further subdivided into inpatient, outpatient, and emergency department costs. All amounts were normalized to 2018 U.S. dollars based on the healthcare component of the Consumer Price Index. ${ }^{40}$ All of the above outcomes were also re-analyzed within a subset of only those claims that included OA-related medications, procedure or diagnosis codes in order to compare OA-related utilization and cost among categories. Because of concerns about confounding due to age, sex and BMI, generalized linear regression models were used to test for statistically significant differences among treatmentbased or pain episode categories, with additional terms to adjust for age category (18-44, 45-64, 65-79 or 80+), sex (male or female) and BMI (< or $\geq 30 \mathrm{~kg} / \mathrm{m}^{2}$ ). These models used a binomial distribution for frequency of utilization, normal distribution for rates of utilization, and log-link function and gamma distribution for costs. Results were expressed as estimates of the utilization and cost for a reference group of females age $45-64$ with BMI $\geq 30$. All statistical analysis was performed using SAS software (SAS 9.4, Cary, NC).

Table 1. Treatment-based severity criteria used to define moderate-severe category of OA.

\begin{tabular}{|c|c|c|}
\hline Name & Description & References \\
\hline Procedures & $\begin{array}{l}\text { At least } 1 \text { OA-related hip or knee surgery, including knee } \\
\text { injections }\end{array}$ & $\begin{array}{l}\text { Meneses et al., } \\
\mid B J^{24,33}\end{array}$ \\
\hline Anxiety/Depression & $\begin{array}{l}\text { At least } 2 \text { encounters with a primary diagnosis of anxiety or } \\
\text { depression, occurring within } 180 \text { days of an encounter with a } \\
\text { primary diagnosis of OA }\end{array}$ & $\begin{array}{l}\text { Deveza et al., White } \\
\text { et al. } 25,34\end{array}$ \\
\hline Opioid & $\begin{array}{l}\text { At least } 2 \text { prescriptions for opioid, each occurring within } 90 \\
\text { days of an encounter with primary diagnosis of OA }\end{array}$ & $\begin{array}{l}\text { Menseses et al., } \\
\text { IBJI, NICE (2019), } \\
\text { Hochberg et } \\
\text { al. }^{24,26,33,35}\end{array}$ \\
\hline NSAID & $\begin{array}{l}\text { At least } 2 \text { prescriptions for nonsteroidal anti-inflammatory } \\
\text { drugs (NSAID) each occurring within } 90 \text { days of an encounter } \\
\text { with primary diagnosis of OA }\end{array}$ & $\begin{array}{l}\text { Menseses et al., } \\
\text { IBJI, NICE (2019), } \\
\text { Hochberg et } \\
\text { al. }^{24,26,33,35}\end{array}$ \\
\hline $\begin{array}{l}\text { HA or IA } \\
\text { Corticosteroids }\end{array}$ & $\begin{array}{l}\text { At least } 2 \text { administrations of hyaluronic acid }(\mathrm{HA}) \text { or intra- } \\
\text { articular }(\mathrm{IA}) \text { corticosteroids, at least } 90 \text { days apart }\end{array}$ & $\begin{array}{l}\text { Meneses et al., } \\
\text { Hochberg et al. } 24,26\end{array}$ \\
\hline Mobility Aid & $\begin{array}{l}\text { At least } 1 \text { prescription order for a mobility aid (e.g., walker) } \\
\text { occurring within } 30 \text { days of an encounter with primary } \\
\text { diagnosis of OA }\end{array}$ & * \\
\hline $\begin{array}{l}\text { Physical/Occupational } \\
\text { Therapy }\end{array}$ & $\begin{array}{l}\text { At least } 1 \text { referral to, or completed encounter with, a physical } \\
\text { or occupational therapy department, occurring within } 30 \text { days } \\
\text { of an encounter with primary diagnosis of OA }\end{array}$ & $\begin{array}{l}\text { Arthritis Foundation, } \\
\text { NICE }(2014)^{36,37}\end{array}$ \\
\hline X-Rays & $\begin{array}{l}\text { At least } 2 \text { X-ray examinations (excluding chest X-rays) }<365 \\
\text { days apart, each occurring within } 90 \text { days of an encounter } \\
\text { with primary diagnosis of OA }\end{array}$ & * \\
\hline
\end{tabular}

*Based on expert opinion from the study team, no specific reference. 
There were 290,897 patients identified in the EHR with a diagnosis of OA during the study period; 127,656 (44\%) of these had at least 1 month of claims information available after OA diagnosis and were included in the study. Of the 127,656 patients, approximately $26 \%$ progressed from mild to moderate-severe OA during the study and therefore contributed to both categories, while $48 \%$ were only mild and $26 \%$ were only observed after progressing to moderate-severe disease. Of the patients eligible for the study, 92,576 had at least one pain episode, for a total of 306,200 pain episodes available for analysis ( $43 \%$ mild, $32 \%$ moderate and $25 \%$ severe).

Baseline demographics of insured OA patients included in this study were similar to those of our health system's OA population overall. Table 2 presents columns showing the baseline characteristics of the initially-identified EHR population ( $n=290,897)$, those with insurance claims who were eligible for the current study $(n=127,656)$, and those with insurance claims and pain episodes who were eligible for the pain-based analysis $(n=92,576)$. The initial OA population had a mean age of 50 , with $59 \%$ females, $97 \%$ white/Caucasian and $97 \%$ non-Hispanic (reflecting the geographic region), $54 \%$ obese (body mass index $\geq 30$ ), and a mean $\mathrm{CCl}$ of 1.0 , similar to both of the subgroups analyzed.

Patients divided into treatment-based categories and pain episodes did show differences in baseline characteristics, as shown in Table 3. Patients with moderate-severe OA were older than those with mild OA (mean age 57 vs. 49 years), less likely to have a $\mathrm{BMI}<30$ (39\% vs. $50 \%)$, and more likely to have a $\mathrm{BMI}>35(27 \%$ vs. $20 \%)$. Patients experiencing moderate and severe pain episodes had a lower mean age than those in mild pain episodes (54 vs. 54 vs. 57 years, respectively) and were more likely to be female (63\% vs. $65 \%$ vs. $59 \%$, respectively). Charlson Comorbidity Index was similar among groups. The regression adjustment for age, sex and BMI was utilized when comparing utilization and cost, therefore, to address these possible sources of confounding.

Moderate and severe pain episodes were associated with statistically significantly higher frequencies and rates of every category of OA-related utilization when compared with mild pain episodes (OP visits: 1.01 vs. 1.61 vs. 1.83 PMPY for mild, moderate and severe pain, respectively; ED visits: 0.14 vs. 0.27 vs. 0.47 PMPY, respectively; IP days: 0.18 vs. 0.40 vs. 0.42 ; all p-values $<0.0001$ ). All-cause ED frequency and visits PMPY also significantly increased for severe pain vs. mild pain, but all other categories of all-cause utilization decreased or stayed the same with increasing pain severity (OP visits: 14.1 vs. 13.6 vs. 14.0 ; ED visits: 1.47 vs. 1.38 vs. 2.03 ; IP days: 4.7 vs. 3.8 vs. 3.3 ). Similarly, we observed significant increases in every category of OA-related costs during moderate and severe pain episodes when compared to mild pain (for example, pharmacy: \$729 vs. \$961 vs. \$1,228 PMPY for mild, moderate, and severe, respectively; medical: $\$ 1,505$ vs. $\$ 2,613$ vs. $\$ 2,742$; all p-values $<0.0001)$. In contrast, pharmacy was the only type of all-cause cost that was positively associated with increasing pain severity (pharmacy: $\$ 9,114$ vs. $\$ 9,558$ vs. \$10,591), while all other all-cause costs decreased with increasing pain. These data are presented in Figure 1 and Table 4.

All OA-related utilization and costs were significantly higher in both frequency and PMPY rates for patients in the moderate-severe OA category when compared to mild OA (OP visits: 0.58 vs. 1.08 PMPY for mild vs. moderatesevere OA, respectively; ED visits: 0.11 vs. 0.17 ; IP days: 0.07 vs. 0.24 ; pharmacy cost: $\$ 543$ vs. $\$ 825$; medical cost: $\$ 576$ vs. $\$ 1,985$; all p-values $<0.0001)$. Patients in the moderate-severe OA category also had statistically significantly higher rates of all-cause ED visits and OP visits than mild OA patients (ED visits: 0.77 vs. 0.86 for mild vs. moderate-severe; OP visits: 9.7 vs. 10.0) but a similar rate of all-cause IP days (2.3 vs. 2.2, $p=0.53)$. Pharmacy and ED were the only two costs that were significantly higher for moderate-severe OA compared with mild OA (pharmacy: \$6,127 vs. \$7,707; ED; \$715 vs. \$786), while all other all-cause cost categories were significantly lower 
for moderate-severe OA patients. All significant $p$-values noted above were $<0.0001$. These data are presented in Figure 2 and Table 5.

Finally, the secondary analysis comparing prevalence of the eight treatment-based severity criteria between incident OA patients with TKR and patients without joint replacement showed that all criteria displayed statistically significant risk ratios greater than 1.0. Because the former patients were more likely to have met these criteria before TKR surgery than other OA patients were to have met them before the study period ended, these data provide additional empirical support for these treatment-based criteria, which were largely based on guideline and expert opinion. These comparisons and risk ratios are shown in Table 6.

Table 2. Patient demographics of the initially identified OA population, OA patients with $>30$ days of insurance claims, and $O A$ patients with $>30$ days of insurance claims and $\geq 1$ pain episode. Characteristics reflect the patients as of index date, unless otherwise noted. 


\begin{tabular}{|c|c|c|c|}
\hline & $\begin{array}{l}\text { All OA } \\
\text { patients in } \\
\text { EHR } \\
(n=290897)\end{array}$ & $\begin{array}{l}\text { With }>30 \text { days of } \\
\text { insurance enrollment } \\
(n=127656)\end{array}$ & $\begin{array}{l}\text { With }>30 \text { days insurance enrollment } \\
\text { and } \geq 1 \text { pain episode }(n=92576)\end{array}$ \\
\hline \multicolumn{4}{|l|}{ Age, in years, $N(\%)$} \\
\hline $18-44$ & \multirow{2}{*}{$\begin{array}{l}111401 \\
(38 \%)\end{array}$} & $55055(43 \%)$ & 39918 (43\%) \\
\hline $45-64$ & & $48831(38 \%)$ & 36054 (39\%) \\
\hline $65-79$ & $\begin{array}{l}108214 \\
(37 \%)\end{array}$ & 20637 (16\%) & $14953(16 \%)$ \\
\hline $80+$ & \multirow{3}{*}{$\begin{array}{l}56361 \\
(19 \%) \\
14921(5 \%) \\
50.5(18.5)\end{array}$} & $3133(2 \%)$ & $1651(2 \%)$ \\
\hline Mean (SD) & & $47.7(17.7)$ & $47.5(17.2)$ \\
\hline & & & \\
\hline \multicolumn{4}{|l|}{ Sex, N (\%) } \\
\hline Males & \multirow{2}{*}{$\begin{array}{l}120207 \\
(41 \%) \\
170662 \\
(59 \%)\end{array}$} & $51077(40 \%)$ & $36535(39 \%)$ \\
\hline Females & & $76563(60 \%)$ & $56031(61 \%)$ \\
\hline \multicolumn{4}{|l|}{ Race, N (\%) } \\
\hline White/Caucasian & \multirow{4}{*}{$\begin{array}{l}280868 \\
(97 \%) \\
6985(2 \%) \\
1353(<1 \%) \\
1691(<1 \%)\end{array}$} & $123788(97 \%)$ & 89807 (97\%) \\
\hline African American & & $2624(2 \%)$ & $1910(2 \%)$ \\
\hline Asian & & $588(<1 \%)$ & $427(<1 \%)$ \\
\hline Other & & $656(<1 \%)$ & $432(<1 \%)$ \\
\hline \multicolumn{4}{|l|}{ Ethnicity, N (\%) } \\
\hline Non-Hispanic & \multirow{2}{*}{$\begin{array}{l}283227 \\
(97 \%) \\
7670(3 \%)\end{array}$} & $124165(97 \%)$ & 89940 (97\%) \\
\hline Hispanic & & 3491 (3\%) & $2636(3 \%)$ \\
\hline \multicolumn{4}{|c|}{ Body mass index (BMI) in } \\
\hline$<30$ & $\begin{array}{l}132722 \\
(46 \%)\end{array}$ & $31078(24 \%)$ & 22846 (25\%) \\
\hline $30-35$ & $\begin{array}{l}68371 \\
(24 \%)\end{array}$ & $25381(20 \%)$ & $18762(20 \%)$ \\
\hline $\begin{array}{l}>35 \\
\text { Unknown }\end{array}$ & $\begin{array}{l}54819 \\
(19 \%)\end{array}$ & $12901(10 \%)$ & $8854(10 \%)$ \\
\hline & $\begin{array}{l}34985 \\
(12 \%)\end{array}$ & & \\
\hline
\end{tabular}




\begin{tabular}{|c|c|c|c|}
\hline & $\begin{array}{l}\text { All OA } \\
\text { patients in } \\
\text { EHR } \\
(n=290897)\end{array}$ & $\begin{array}{l}\text { With }>30 \text { days of } \\
\text { insurance enrollment } \\
(n=127656)\end{array}$ & $\begin{array}{l}\text { With }>30 \text { days insurance enrollment } \\
\text { and } \geq 1 \text { pain episode }(n=92576)\end{array}$ \\
\hline \multicolumn{4}{|l|}{ Smoking status, $\mathbf{N}(\%)$} \\
\hline Never & $\begin{array}{l}133576 \\
(46 \%)\end{array}$ & $55683(44 \%)$ & $40385(44 \%)$ \\
\hline Current & 52865 & $26968(21 \%)$ & $20121(22 \%)$ \\
\hline Quit & $\begin{array}{l}52800 \\
(18 \%)\end{array}$ & 29911 (23\%) & 22928 (25\%) \\
\hline \multirow[t]{2}{*}{ Unknown/Not Asked } & $\begin{array}{l}44733 \\
(15 \%)\end{array}$ & $15094(12 \%)$ & $9142(10 \%)$ \\
\hline & $\begin{array}{l}59723 \\
(21 \%)\end{array}$ & & \\
\hline \multicolumn{4}{|l|}{$\begin{array}{l}\text { Charlson Comorbidity Index } \\
\text { (CCI) }\end{array}$} \\
\hline Mean (SD) & $1.0(1.8)$ & $0.9(1.6)$ & $0.9(1.7)$ \\
\hline With $\mathrm{CCl}>0, \mathrm{~N}(\%)$ & $\begin{array}{l}115615 \\
(40 \%)\end{array}$ & $48619(38 \%)$ & $37013(40 \%)$ \\
\hline \multicolumn{4}{|l|}{$\begin{array}{l}\text { Insurance Coverage during } \\
\text { study, N (\%) }\end{array}$} \\
\hline $\begin{array}{l}>5 \text { years of claims in } \\
\text { affiliated health plan, } N(\%)\end{array}$ & $\begin{array}{l}85848 \\
(30 \%)\end{array}$ & 41051 (32\%) & 35375 (38\%) \\
\hline \multicolumn{4}{|l|}{$\begin{array}{l}\text { Joint replacement surgery } \\
\text { during study, } N(\%)\end{array}$} \\
\hline $\begin{array}{l}\text { With total knee replacement } \\
\text { (TKR) }\end{array}$ & $\begin{array}{l}10780(4 \%) \\
6359(2 \%)\end{array}$ & $\begin{array}{l}5796(5 \%) \\
3076(2 \%)\end{array}$ & $\begin{array}{l}4989(5 \%) \\
2622(3 \%)\end{array}$ \\
\hline \multicolumn{4}{|l|}{$\begin{array}{l}\text { With total hip replacement } \\
\text { (THR) }\end{array}$} \\
\hline \multicolumn{4}{|l|}{$\begin{array}{l}\text { Treatment intensity level } \\
\text { information }\end{array}$} \\
\hline Mild only & $\begin{array}{l}157690 \\
(54 \%)\end{array}$ & $61158(48 \%)$ & $41892(45 \%)$ \\
\hline $\begin{array}{l}\text { Progressed from mild to } \\
\text { moderate-severe }\end{array}$ & $\begin{array}{l}70323 \\
(24 \%)\end{array}$ & $\begin{array}{l}33143(26 \%) \\
33355(26 \%)\end{array}$ & $\begin{array}{l}25963(28 \%) \\
24721(27 \%)\end{array}$ \\
\hline Moderate-severe only & $\begin{array}{l}62884 \\
(22 \%)\end{array}$ & & \\
\hline
\end{tabular}

Table 3. Baseline characteristics compared among treatment-based and pain categories, as of the patient's first observation in each category. Standardized difference $|\mathrm{d}|$ is used here to assess balance, with $|\mathrm{d}|>0.10$ (in bold) suggesting imbalances in age and BMI between treatment-based categories and imbalances in age and sex among pain categories. 


\begin{tabular}{|c|c|c|c|c|c|c|c|c|}
\hline & \multicolumn{5}{|c|}{ PAIN EPISODE CATEGORY } & \multicolumn{3}{|c|}{$\begin{array}{l}\text { TREATMENT-BASED } \\
\text { CATEGORY }\end{array}$} \\
\hline & $\begin{array}{l}\text { Mild } \\
(\mathrm{n}=61823)\end{array}$ & $\begin{array}{l}\text { Moderate } \\
(n=52208)\end{array}$ & $\begin{array}{l}\text { Severe } \\
(n=40303)\end{array}$ & $\begin{array}{l}|\mathrm{d}| \\
\text { Moderate } \\
\text { vs. Mild }\end{array}$ & $\begin{array}{l}|d| \\
\text { Severe } \\
\text { vs. } \\
\text { Mild }\end{array}$ & $\begin{array}{l}\text { Mild } \\
(\mathrm{n}=83625)\end{array}$ & $\begin{array}{l}\text { Moderate- } \\
\text { Severe } \\
(n=63615)\end{array}$ & $|d|$ \\
\hline \multicolumn{9}{|l|}{$\begin{array}{l}\text { Age, in } \\
\text { years, } \mathrm{N}(\%)\end{array}$} \\
\hline $18-44$ & $\begin{array}{l}14268 \\
(23 \%)\end{array}$ & $\begin{array}{l}15311 \\
(29 \%)\end{array}$ & $\begin{array}{l}11904 \\
(30 \%)\end{array}$ & $\begin{array}{l}0.14 \\
0.02\end{array}$ & 0.15 & $\begin{array}{l}33791 \\
(40 \%)\end{array}$ & $\begin{array}{l}12128 \\
(19 \%)\end{array}$ & $\begin{array}{l}0.48 \\
0.20\end{array}$ \\
\hline $45-64$ & $\begin{array}{l}23754 \\
(38 \%)\end{array}$ & $\begin{array}{l}20617 \\
(39 \%)\end{array}$ & $\begin{array}{l}15896 \\
(39 \%)\end{array}$ & 0.11 & $\begin{array}{l}0.02 \\
0.13\end{array}$ & $\begin{array}{l}31306 \\
(37 \%)\end{array}$ & $\begin{array}{l}29979 \\
(47 \%)\end{array}$ & 0.21 \\
\hline $80+$ & $\begin{array}{l}17300 \\
(28 \%)\end{array}$ & $\begin{array}{l}12001 \\
(23 \%)\end{array}$ & $\begin{array}{l}8952 \\
(22 \%)\end{array}$ & \multirow{3}{*}{$\begin{array}{l}0.08 \\
0.17\end{array}$} & \multirow{2}{*}{$\begin{array}{l}0.06 \\
0.17\end{array}$} & $\begin{array}{l}14903 \\
(18 \%)\end{array}$ & $\begin{array}{l}16879 \\
(27 \%)\end{array}$ & 0.13 \\
\hline \multirow{2}{*}{$\begin{array}{l}\text { Mean } \\
\text { (SD) }\end{array}$} & $\begin{array}{l}6501 \\
(11 \%)\end{array}$ & $4279(8 \%)$ & 3551 (9\%) & & & $3625(4 \%)$ & $4629(7 \%)$ & \multirow{2}{*}{0.47} \\
\hline & $57(18)$ & $54(18)$ & $54(18)$ & & & $49(18)$ & $57(16)$ & \\
\hline \multicolumn{9}{|l|}{$\operatorname{Sex}, N(\%) *$} \\
\hline \multirow{2}{*}{$\begin{array}{l}\text { Males } \\
\text { Females }\end{array}$} & $\begin{array}{l}25304 \\
(41 \%)\end{array}$ & $\begin{array}{l}19335 \\
(37 \%)\end{array}$ & $\begin{array}{l}13987 \\
(35 \%)\end{array}$ & \multirow[t]{2}{*}{0.08} & \multirow[t]{2}{*}{0.13} & $\begin{array}{l}33414 \\
(40 \%)\end{array}$ & $\begin{array}{l}25094 \\
(39 \%)\end{array}$ & \multirow[t]{2}{*}{0.01} \\
\hline & $\begin{array}{l}36511 \\
(59 \%)\end{array}$ & $\begin{array}{l}32869 \\
(63 \%)\end{array}$ & $\begin{array}{l}26315 \\
(65 \%)\end{array}$ & & & $\begin{array}{l}50200 \\
(60 \%)\end{array}$ & $\begin{array}{l}38512 \\
(61 \%)\end{array}$ & \\
\hline \multicolumn{9}{|l|}{$\begin{array}{l}\text { Body mass } \\
\text { index (BMI) }\end{array}$} \\
\hline in $\mathrm{kg} / \mathrm{m}^{2}, \mathrm{~N}$ & $\begin{array}{l}30275 \\
(49 \%)\end{array}$ & $\begin{array}{l}24056 \\
(46 \%)\end{array}$ & $\begin{array}{l}18031 \\
(45 \%)\end{array}$ & $\begin{array}{l}0.06 \\
0.00\end{array}$ & $\begin{array}{l}0.08 \\
0.00\end{array}$ & \multirow{2}{*}{$\begin{array}{l}(50 \%) \\
21400 \\
(26 \%)\end{array}$} & $\begin{array}{l}25061 \\
(39 \%)\end{array}$ & \multirow{2}{*}{$\begin{array}{l}0.22 \\
0.08 \\
0.15\end{array}$} \\
\hline$<30$ & $\begin{array}{l}17557 \\
(28 \%)\end{array}$ & $\begin{array}{l}14884 \\
(29 \%)\end{array}$ & $\begin{array}{l}11412 \\
(28 \%)\end{array}$ & 0.06 & 0.09 & & $\begin{array}{l}18555 \\
(29 \%)\end{array}$ & \\
\hline $\begin{array}{l}30-35 \\
>35\end{array}$ & $\begin{array}{l}13757 \\
(22 \%)\end{array}$ & $\begin{array}{l}12921 \\
(2 \% \%)\end{array}$ & $\begin{array}{l}10603 \\
(26 \%)\end{array}$ & \multirow[t]{2}{*}{0.04} & 0.04 & $\begin{array}{l}16944 \\
(20 \%)\end{array}$ & $\begin{array}{l}17019 \\
(27 \%)\end{array}$ & 0.03 \\
\hline Unknown & $234(<1 \%)$ & $347(<1 \%)$ & $257(<1 \%)$ & & & $3424(4 \%)$ & $2980(5 \%)$ & \\
\hline $\begin{array}{l}\text { Charlson } \\
\text { Comorbidity } \\
\text { Index (CCI) }\end{array}$ & $\begin{array}{l}0.97 \\
(1.71)\end{array}$ & $\begin{array}{l}0.84 \\
(1.54)\end{array}$ & $\begin{array}{l}0.82 \\
(1.49)\end{array}$ & 0.08 & 0.09 & $\begin{array}{l}0.89 \\
(1.68)\end{array}$ & $\begin{array}{l}0.79 \\
(1.42)\end{array}$ & 0.06 \\
\hline $\begin{array}{l}\text { Mean (SD) } \\
\text { With CCl > } \\
0, N(\%)\end{array}$ & $\begin{array}{l}25517 \\
(41 \%)\end{array}$ & $\begin{array}{l}20383 \\
(39 \%)\end{array}$ & $\begin{array}{l}15837 \\
(39 \%)\end{array}$ & 0.05 & 0.04 & $\begin{array}{l}32007 \\
(38 \%)\end{array}$ & $\begin{array}{l}24256 \\
(38 \%)\end{array}$ & 0.00 \\
\hline
\end{tabular}

*Sex was not reported for 33 patients, so totals do not sum to $100 \%$. SD = standard deviation

Table 4. Frequency and units of utilization, and costs compared among the three pain episode types. Estimates and p-values from generalized linear regression models are adjusted for age, sex and BMI, and estimates reflect a reference group of females with age 45-64 and $\mathrm{BMI}>30$. P-values in bold reflect significant decreases in utilization or cost between moderate vs. mild or severe vs. mild pain episodes, while p-values in italics reflect significant increases. 
p-values

$\begin{array}{lllll}\text { Mild } & \text { Moderate } & \text { Severe } & \begin{array}{l}\text { Moderate vs } \\ \text { Mild }\end{array} & \begin{array}{l}\text { Severe vs } \\ \text { Mild }\end{array} \\ (n=61823) & (n=52208) & (n=40303) & & \end{array}$

\section{All-cause resource use}

Frequency of use, $\mathrm{N}$

(\%)

\begin{tabular}{llllll} 
ED visits & $\begin{array}{l}27388 \\
(44.3 \%)\end{array}$ & $\begin{array}{l}21666 \\
(41.5 \%)\end{array}$ & $\begin{array}{l}25403 \\
(50.5 \%)\end{array}$ & $<.0001$ & $<.0001$ \\
& & & & \\
Outpatient visits & 58608 & 48919 & 47687 & $<.92$ \\
& $(94.8 \%)$ & $(93.7 \%)$ & $(94.8 \%)$ & $<.0001$ & $<.0001$ \\
\hline \multirow{2}{*}{ Inpatient admissions } & 14776 & 11329 & 11117 & $<.0001$ & \\
& $(23.9 \%)$ & $(21.7 \%)$ & $(22.1 \%)$ & &
\end{tabular}

Units PMPY, Mean (CI)

\begin{tabular}{lllllc} 
ED visits & $1.47(0.04)$ & $1.38(0.04)$ & $2.03(0.04)$ & $<.0001$ & $<.0001$ \\
\hline Outpatient visits & $14.1(0.15)$ & $13.6(0.15)$ & $14.0(0.16)$ & $<.0001$ & 0.52 \\
\hline Inpatient days & $4.7(0.2)$ & $3.8(0.2)$ & $3.3(0.3)$ & $<.0001$ & $<.0001$
\end{tabular}

Costs PMPY, Mean (CI)

$S$

\begin{tabular}{llllll} 
Total & $37713(537)$ & $32739(369)$ & $31334(477)$ & $<.0001$ & $<.0001$ \\
Pharmacy & $9114(194)$ & $9558(203)$ & $10591(239)$ & $<.0001$ & $<.0001$ \\
Medical & $28357(458)$ & $23394(384)$ & $21264(371)$ & $<.0001$ & $<.0001$ \\
\hline Inpatient & $12539(457)$ & $10431(378)$ & $10077(390)$ & $<.0001$ & $<.0001$ \\
Outpatient & $13379(245)$ & $9873(183)$ & $8441(168)$ & $<.0001$ & $<.0001$ \\
ED & $2251(64)$ & $2014(59)$ & $2131(65)$ & $<.0001$ & 0.0004
\end{tabular}

\section{OA-related resource use}

Frequency of use, $N$

(\%)

\begin{tabular}{|lllllr}
\hline ED visits & $6677(10.8 \%)$ & $7670(14.5 \%)$ & $\begin{array}{l}10715 \\
(21.3 \%)\end{array}$ & $<.0001$ & $<.0001$ \\
\hline Outpatient visits & $\begin{array}{l}20216 \\
(32.7 \%)\end{array}$ & $\begin{array}{l}23807 \\
(45.6 \%)\end{array}$ & $\begin{array}{l}25705 \\
(51.1 \%)\end{array}$ & $<.0001$ & $<.0001$ \\
\hline Inpatient admissions & $1731(2.8 \%)$ & $2976(5.7 \%)$ & $3219(6.4 \%)$ & $<.0001$ & $<.0001$ \\
\hline Units PMPY, Mean (CI) & & & & & $<.0001$ \\
\hline ED visits & $0.14(0.01)$ & $0.27(0.01)$ & $0.47(0.01)$ & $<.0001$ & $<.0001$ \\
\hline Outpatient visits & $1.01(0.03)$ & $1.61(0.03)$ & $1.83(0.03)$ & $<.0001$ & $<.0001$ \\
\hline Inpatient days & $0.18(0.03)$ & $0.40(0.03)$ & $0.42(0.03)$ & $<.0001$ &
\end{tabular}




\begin{tabular}{|c|c|c|c|c|c|}
\hline Total & $2209(50)$ & 3629 (85) & 4110 (103) & $<.0001$ & $<.0001$ \\
\hline Pharmacy & 729 (18) & $961(24)$ & $1228(32)$ & $<.0001$ & $<.0001$ \\
\hline Medical & $1505(42)$ & $2613(74)$ & 2742 (83) & $<.0001$ & $<.0001$ \\
\hline Inpatient & $1048(37)$ & 2023 (72) & 2144 (83) & $<.0001$ & $<.0001$ \\
\hline Outpatient & $595(17)$ & $708(21)$ & 751 (23) & $<.0001$ & $<.0001$ \\
\hline ED & $49(2)$ & $63(2)$ & $96(3)$ & $<.0001$ & $<.0001$ \\
\hline
\end{tabular}

$\mathrm{BMI}=$ body mass index, $\mathrm{ED}=$ emergency department, $\mathrm{PMPY}=$ per member per year, $\mathrm{OA}=$ osteoarthritis, $\mathrm{Cl}=\mathrm{half}-$ width of the $95 \%$ confidence interval ( \pm 2 standard errors of the mean)

Table 5. Frequency and units of utilization, and costs, compared among the two treatment-based severity levels. Estimates and $p$-values are adjusted for age, sex and BMI, and estimates reflect a reference group of females with age 45-64 and BMl>30. P-values in bold reflect decreases in utilization or cost between moderate-severe vs. mild OA, and p-values in italics reflect increases in utilization or cost. 


\begin{tabular}{|c|c|c|c|}
\hline & $\begin{array}{l}\text { Mild } \\
(n=83625)\end{array}$ & $\begin{array}{l}\text { Moderate-Severe } \\
(n=63615)\end{array}$ & p-value \\
\hline \multicolumn{4}{|l|}{ All-cause resource use } \\
\hline \multicolumn{4}{|l|}{ Frequency of use, $N(\%)$} \\
\hline ED visits & $37799(45.2 \%)$ & $38360(60.3 \%)$ & $<.0001$ \\
\hline Outpatient visits & $79611(95.2 \%)$ & $61897(97.3 \%)$ & $<.0001$ \\
\hline Inpatient admissions & $22328(26.7 \%)$ & $26146(41.1 \%)$ & $<.0001$ \\
\hline \multicolumn{4}{|l|}{ Units PMPY, Mean $(C l)$} \\
\hline ED visits & $0.77(0.006)$ & $0.86(0.03)$ & $<.0001$ \\
\hline Outpatient visits & $9.7(0.11)$ & $10.0(0.11)$ & $<.0001$ \\
\hline Inpatient days & $2.3(0.14)$ & $2.2(0.13)$ & 0.53 \\
\hline \multicolumn{4}{|l|}{ Costs PMPY, Mean $(C I) S$} \\
\hline Total & $23027(330)$ & $21023(290)$ & $<.0001$ \\
\hline Pharmacy & $6127(131)$ & 7707 (159) & $<.0001$ \\
\hline Medical & $16816(262)$ & $13424(203)$ & $<.0001$ \\
\hline Inpatient & $5174(167)$ & $4763(146)$ & $<.0001$ \\
\hline Outpatient & $7793(135)$ & $5225(88)$ & $<.0001$ \\
\hline ED & $715(18)$ & $786(19)$ & $<.0001$ \\
\hline \multicolumn{4}{|l|}{ OA-related resource use } \\
\hline \multicolumn{4}{|l|}{ Frequency of use, $N(\%)$} \\
\hline ED visits & $12962(15.5 \%)$ & $18894(29.7 \%)$ & $<.0001$ \\
\hline Outpatient visits & $33952(40.6 \%)$ & $45676(71.8 \%)$ & $<.0001$ \\
\hline Inpatient admissions & $920(1.1 \%)$ & $10306(16.2 \%)$ & $<.0001$ \\
\hline \multicolumn{4}{|l|}{ Units PMPY, Mean (Cl) } \\
\hline ED visits & $0.11(0.007)$ & $0.17(0.007)$ & $<.0001$ \\
\hline Outpatient visits & $0.58(0.02)$ & $1.08(0.02)$ & $<.0001$ \\
\hline Inpatient days & $0.07(0.02)$ & $0.24(0.02)$ & $<.0001$ \\
\hline \multicolumn{4}{|l|}{ Costs PMPY, Mean (CI) $\$$} \\
\hline Total & $1137(23)$ & 2801 (54) & $<.0001$ \\
\hline Pharmacy & $543(13)$ & $825(19)$ & $<.0001$ \\
\hline Medical & $576(13)$ & 1985 (45) & $<.0001$ \\
\hline Inpatient & $182(6)$ & $1184(36)$ & $<.0001$ \\
\hline
\end{tabular}




\begin{tabular}{|llll|}
\hline Outpatient & $197(5)$ & $460(11)$ & $<.0001$ \\
\hline ED & $21(0.5)$ & $30(0.7)$ & $<.0001$ \\
\hline
\end{tabular}

$\mathrm{BMI}=$ body mass index, $\mathrm{ED}=$ emergency department, $\mathrm{PMPY}=$ per member per year, $\mathrm{OA}=$ osteoarthritis, $\mathrm{Cl}=$ halfwidth of the $95 \%$ confidence interval ( \pm 2 standard errors of the mean)

Table 6. Numbers and percentages of patients in two subgroups (incident OA +TKR, or no THR or TKR) meeting each of the eight criteria used in this study to define moderate-severe OA, measured as of 180 days before their TKR or last encounter. Relative risk ratios were all positive and $95 \%$ confidence intervals did not overlap 1.0, indicating that incident OA patients with TKR were significantly more likely than other group to have met all criteria, at the $p<0.05$ significance level.

\begin{tabular}{|llll|}
\hline $\begin{array}{l}\text { Patients meeting criteria, } \\
\text { ( } \%)\end{array}$ & $\begin{array}{l}\text { Incident OA Patients } \\
\text { with TKR } \\
(\mathbf{n = 4 2 5 6 )}\end{array}$ & $\begin{array}{l}\text { Patients with no THR } \\
\text { or TKR } \\
(\mathbf{n = 2 7 4 4 8 9 )}\end{array}$ & $\begin{array}{l}\text { Relative Risk Ratio } \\
\mathbf{( 9 5 \% ~ C l )} \\
\text { (Incident OA with TKR vs. No } \\
\text { TKR/THR) }\end{array}$ \\
\hline Procedures & $2121(50 \%)$ & $59574(22 \%)$ & $2.30(2.23,2.37)$ \\
\hline Anxiety/Depression & $929(22 \%)$ & $36500(13 \%)$ & $1.64(1.55,1.73)$ \\
\hline Opioid & $1643(39 \%)$ & $46676(17 \%)$ & $2.27(2.18,2.36)$ \\
\hline NSAID & $1286(30 \%)$ & $31227(11 \%)$ & $2.66(2.53,2.79)$ \\
\hline HA or IA Corticosteroids & $1090(26 \%)$ & $11000(4 \%)$ & $6.39(6.06,6.72)$ \\
\hline Mobility Aid & $81(2 \%)$ & $3208(1 \%)$ & $1.63(1.26,1.99)$ \\
\hline $\begin{array}{l}\text { Physical/Occupational } \\
\text { Therapy }\end{array}$ & $288(7 \%)$ & $10469(4 \%)$ & $1.77(1.57,1.97)$ \\
\hline X-Rays & $2853(67 \%)$ & $58275(21 \%)$ & $3.16(3.09,3.22)$ \\
\hline
\end{tabular}

$\mathrm{OA}=$ osteoarthritis, $\mathrm{TKR}=$ total knee replacement, $\mathrm{THR}=$ total hip replacement, $\mathrm{TJR}=$ total joint replacement, NSAID = nonsteroidal anti-inflammatory drug, $\mathrm{HA}=$ hyaluronic acid, $\mathrm{IA}=$ intra-articular

\section{Discussion}

This study examined utilization and cost differences among subgroups of OA patients, segmented into groups using two different approaches. One approach was based on patients meeting one of eight treatment-based criteria indicating transition from mild to moderate-severe $\mathrm{OA}$, and the other approach was based on shorter periods of time (90 days or more) triggered by a pain score in the mild, moderate, or severe range. Analyses adjusting for age, sex and BMI differences showed increases in utilization and costs in every OA-related category with increasing severity, using either a treatment-based or pain-based severity definition. Some similar associations were observed with allcause resource utilization and costs, but that evidence was much more mixed, with many all-cause utilization and cost types that decreased as pain or treatment severity increased. 
We saw increases in every subcategory of OA-related utilization and costs for patients who were defined as having more severe $\mathrm{OA}$, whether that severity was defined by the treatment intensity or pain severity. Given the importance of pain as a symptom of $\mathrm{OA}$, these associations have strong face validity and are consistent with what has been shown in other OA populations. Wei et al. ${ }^{21}$, which used claims from 35,861 commercial and Medicare Part D knee/hip OA patients in the Optum database, reported statistically significant increases in OA-related costs stratified by pain severity; however, they also saw approximately $16-28 \%$ increases in total costs within each of these pain categories when comparing patients with versus without routine opioid use. Our work expands on that prior work by applying both pain severity and additional treatment criteria (instead of opioid use only), with very comparable results.

These increases in OA-related utilization and cost did not always translate, however, to increases in all-cause (i.e., including non-OA-related) utilization or costs, particularly when classifying the population on the basis of pain score. While treatment-based severity level tracked very well with most types of all-cause utilization, pharmacy and ED were the only types of all-cause costs associated with treatment severity level, and pharmacy was the only type of cost that showed a relationship with pain score severity. This lack of association remains an unexpected finding, though we suggest there are several possible explanations that could be explored further. Predominantly, it is reasonable to hypothesize that pain level may still be getting confounded with treatment; for example, lower pain scores could mean the patient is being treated more aggressively to control the pain and would therefore be incurring higher costs despite a lower reported pain score. Some types of utilization may taper off once the patient has progressed to a worse level; for example, if they have had a procedure or surgery that has helped them, or if they experienced unwanted side effects or other barriers to medication adherence, they may be spending or utilizing less despite the fact that they are further along in the progression of OA overall. We note also that in some prior studies, mean differences in ED visits, outpatient visits and inpatient days between severe vs. mild pain that were statistically significant were still relatively small in clinical magnitude (e.g., only approximately 1.1, 1.9 and 0.8 per patient per year, respectively). ${ }^{21}$

Prior studies have aimed to identify clinically relevant phenotypes for $\mathrm{OA}^{25}$ and Van Spil et al. described a consensus-based framework for conducting and reporting such studies. ${ }^{41}$ There is still, however, no standard set of classification criteria, and this investigation provides further quantitative evidence supporting criteria based on both pain and clinician-ordered treatments. In the 2019 ACR guidelines for OA of the hand, hip and knee, no hierarchy of recommended treatments is provided that would indicate varying levels of severity, and treatments may be used and reused at various times during the course of disease. The ESCEO's 2019 consensus statement ${ }^{38}$ provides more of a guidance on three steps of treatment that may correspond more closely to the progression and severity of the disease, but all steps are based on medications only and no other domains, while the Osteoarthritis Research Society International (OARSI) published its own 2019 treatment guidelines that included non-pharmacological "core" interventions including exercise and education. ${ }^{42}$ Deveza et al.'s 2017 systematic review (and 2019 narrative review) of knee OA phenotype studies noted that few studies combined data from different domains, despite the fact that evidence from different authors showed "pain sensitization, psychological distress, radiographic severity, BMI, muscle strength, inflammation and comorbidities" helped to differentiate OA patients. We did not directly compare our patients' classifications to ESCEO or OARSI classifications or previous authors' knee OA phenotypes, so we cannot assert advantages of our criteria over theirs, but we suggest our work provides further support for the inclusion of non-pharmacologic signals such as concomitant diagnoses and procedures when phenotyping OA patients based on OA disease severity. 
The strength of this study was the novelty of being able to examine a large population over a long timeframe with a combination of both EHR and insurance claims, as opposed to claims only, which previous large studies have often been based on. ${ }^{21}$ We recognize several limitations, however, related to both the pain and treatment-based analyses. We are not able to directly attribute pain severity scores with the patient's $O A$, since the patient could be experiencing pain from other causes. Our study was conducted in a largely Caucasian, non-Hispanic population, and we did not limit our study to hip or knee OA only, so these factors should be considered when directly comparing results with other studies. As this was an observational study, pain scores were not collected at regular intervals in usual practice and so patients vary widely in their number and frequency of scores. We recognize that a functional measure such as the Knee Injury and Osteoarthritis Outcome Score (KOOS) would have been a better outcome than NRS pain, but the study included all types of OA and NRS was the most frequently available outcome in the EHR. Pain is also generally recognized to be a subjective, not objective, measure that could be influenced by other unmeasured factors, and there is uncertainty about the relative timing between when the score was collected and when treatment was administered (e.g., does the pain score reflect a patient's pain before or after a new treatment was given). Nonetheless, we believe this study's results support considering pain when defining OA severity. On the treatment side, we believe this investigation provides even stronger evidence for segmenting patients based on treatments received, with a few caveats. We recognize that caution is needed to avoid circular arguments in which receipt of a treatment (e.g., pain medication) is used as both a classifying variable and an outcome. Our treatmentbased approach, however, assigned patients to the moderate-severe OA category if they received any one of eight types of treatment, none of which would be expected to individually account for the magnitude of differences in utilization or cost seen here. In our results, meeting any one of those criteria was associated with patients significantly more likely to see their OA-related utilization and cost increase in the subsequent period, across all categories, which supports the idea that these individual categories are useful markers to predict future health and economic burden.

\section{Conclusions}

In conclusion, by understanding at what point osteoarthritis patients become greater consumers of healthcare resources, we can deploy targeted preventative strategies aimed at halting progression into the next more costly phase of disease. Previous studies have measured the extent of this burden, particularly with respect to differences between patients with $\mathrm{OA}$ and patients without it, ${ }^{43,44}$ but this is the first study to our knowledge that utilizes patterns of care found in both claims and the EHR to analyze direct financial costs of OA patients in subcategories of disease severity based on both pain and treatment progression, and our results provide promising evidence of better criteria and approaches for predicting disease burden and costs in the future.

\section{Abbreviations}




\begin{tabular}{|c|c|}
\hline ACR & American College of Rheumatology \\
\hline BMI & Body Mass Index \\
\hline $\mathrm{CCl}$ & Charlson Comorbidity Index \\
\hline $\mathrm{Cl}$ & Confidence Interval \\
\hline ED & Emergency Department \\
\hline EHR & Electronic Health Record \\
\hline ESCEO & European Society for Clinical and Economic Aspects of Osteoporosis and Osteoarthritis \\
\hline HA & Hyaluronic Acid \\
\hline IA & Intra-articular \\
\hline ICD-9/10-CM & International Classification of Diseases Ninth/Tenth Revisions, Clinical Modification \\
\hline IP & Inpatient \\
\hline IRB & Institutional Review Board \\
\hline KOOS & Knee Injury and Osteoarthritis Outcome Score \\
\hline NRS & Numerical Rating Score \\
\hline NSAID & Nonsteroidal Anti-Inflammatory Drug \\
\hline OA & Osteoarthritis \\
\hline OARSI & Osteoarthritis Research Society International \\
\hline OP & Outpatient \\
\hline PMPY & Per-Member-Per-Year \\
\hline RRR & Relative Risk Ratios \\
\hline SD & Standard Deviation \\
\hline THR & Total Hip Replacement \\
\hline TKR & Total Knee Replacement \\
\hline US & United States \\
\hline
\end{tabular}

\section{Declarations}

\section{Ethics Approval and Consent to Participate}

This study was reviewed and approved (IRB \#2019-1033) by Geisinger's Institutional Review Board (IRB) as meeting the criteria for exemption as defined in the U. S. Department of Health and Human Services Regulations for the Protection of Human Subjects [(45 CFR 46.104)]. The specific exemption category under 45 CFR 46.104 was category 4 , secondary research for which consent was not required.

\section{Consent for Publication}


Not applicable.

\section{Availability of Data and Materials}

The datasets generated and analyzed during the current study are not publicly available due to patient privacy concerns and ownership by Geisinger but additional deidentified information could be made available from the corresponding author on reasonable request.

\section{Competing Interests}

PS, CB, and EC are employees of Pfizer and have stock options. RR and JH are employees of Eli Lilly and Company and have stock options. JG, BP, JB, MK, VH, and EW are employees of Geisinger, which received institutional funding from Pfizer and Eli Lilly and Company for the study. During the study period, TN and HS were employees of Geisinger, which received institutional funding from Pfizer and Eli Lilly and Company for the study.

\section{Funding}

The study was sponsored by Pfizer and Eli Lilly and Company.

\section{Authors' Contributions}

All authors contributed to the conception and design of the study and interpretation of data, and all authors provided substantial contributions to the final manuscript. JG and HS were directly responsible for the primary analysis of data, and JG as first author was responsible for the initial draft of this manuscript.

\section{Acknowledgements}

The authors wish to thank Matt Gass and Celia Gray for their help with assembling the study dataset and thank Pfizer and Eli Lilly and Company for the institutional grant that funded this work.

\section{References}

1. Zhao X, Shah D, Gandhi K, et al. Clinical, humanistic, and economic burden of osteoarthritis among noninstitutionalized adults in the united states. Osteoarthritis and cartilage. 2019;27(11):1618-1626.

2. Osteoarthritis Research Society International. Osteoarthritis: A serious disease, submitted to the U.S. food and drug administration. 2016. https://oarsi.org/sites/default/files/docs/2016/oarsi_white_paper_oa_serious_disease_121416_1.pdf. Accessed 1 December 2021.

3. Woolf AD, Pfleger B. Burden of major musculoskeletal conditions. Bulletin of the World Health Organization. 2003;81(9):646.

4. Hootman JM, Helmick CG. Projections of US prevalence of arthritis and associated activity limitations. Arthritis \& Rheumatism. 2006;54(1):226-229.

5. Liang L, Moore B, Soni A. Agency for Healthcare Research and Quality. National inpatient hospital costs: The most expensive conditions by payer, 2017. 2020. https://www.hcup-us.ahrq.gov/reports/statbriefs/sb261-MostExpensive-Hospital-Conditions-2017.jsp. Accessed 2 December 2021.

6. Centers for Disease Control and Prevention. The cost of arthritis in US adults. 2020. cdc.gov/arthritis/data_statistics/cost.htm. Accessed 2 December 2021. 
7. Murphy LB, Cisternas MG, Pasta DJ, Helmick CG, Yelin EH. Medical expenditures and earnings losses among US adults with arthritis in 2013. Arthritis care \& research (2010). 2018;70(6):869-876.

8. Bitton R. The economic burden of osteoarthritis. The American journal of managed care. 2009;15(Suppl 8):230235.

9. Johnson V, Hunter DJ. The epidemiology of osteoarthritis. Best Practice \& Research: Clinical Rheumatology. 2014;28(1):5-15.

10. Felson DT, Lawrence RC, Dieppe PA, et al. Osteoarthritis: New insights. part 1: The disease and its risk factors. Annals of Internal Medicine. 2000;133(8):635.

11. Loeser RF, Collins JA, Diekman BO. Ageing and the pathogenesis of osteoarthritis. Nature reviews. Rheumatology. 2016;12(7):412-420.

12. Stürmer T, Günther K, Brenner H. Obesity, overweight and patterns of osteoarthritis: The ulm osteoarthritis study. Journal of Clinical Epidemiology. 2000;53(3):307-313.

13. Felson DT, Anderson JJ, Naimark A, Walker AM, Meenan RF. Obesity and knee osteoarthritis. the framingham study. Annals of internal medicine. 1988;109(1):18.

14. Yusuf E, Nelissen RG, loan-Facsinay A, et al. Association between weight or body mass index and hand osteoarthritis: A systematic review. Annals of the Rheumatic Diseases. 2010;69(4):761-765.

15. Lawrence RC, Felson DT, Helmick CG, et al. Estimates of the prevalence of arthritis and other rheumatic conditions in the united states: Part II. Arthritis \& Rheumatism. 2008;58(1):26-35.

16. Hawker GA, Stewart L, French MR, et al. Understanding the pain experience in hip and knee osteoarthritis - an OARSI/OMERACT initiative. Osteoarthritis and Cartilage. 2008;16(4):415-422.

17. Cedraschi C, Delézay S, Marty M, et al. "Let's talk about OA pain”: A qualitative analysis of the perceptions of people suffering from $\mathrm{OA}$. towards the development of a specific pain OA-related questionnaire, the osteoarthritis symptom inventory scale (OASIS). PloS one. 2013;8(11):e79988.

18. De Williams AC, Davies HTO, Chadury Y. Simple pain rating scales hide complex idiosyncratic meanings. Pain (Amsterdam). 2000;85(3):457-463.

19. Dworkin RH, Turk DC, Farrar JT, et al. Core outcome measures for chronic pain clinical trials: IMMPACT recommendations. Pain (Amsterdam). 2005;113(1):9-19.

20. Love BL, Jensen LA, Schopflocher D, Tsui BC. The development of an electronic database for acute pain service outcomes. Pain research \& management. 2012;17(1):25-30.

21. Wei W, Gandhi K, Blauer-Peterson C, Johnson J. Impact of pain severity and opioid use on health care resource utilization and costs among patients with knee and hip osteoarthritis. Journal of managed care \& specialty pharmacy. 2019;25(9):957-965.

22. Haugen IK, Bøyesen P, Slatkowsky-Christensen B, Sesseng S, van der Heijde D, Kvien TK. Associations between MRI-defined synovitis, bone marrow lesions and structural features and measures of pain and physical function in hand osteoarthritis. Annals of the rheumatic diseases. 2012;71(6):899-904.

23. Zhang W, Doherty M, Leeb BF, et al. EULAR evidence-based recommendations for the diagnosis of hand osteoarthritis: Report of a task force of ESCISIT. Annals of the Rheumatic Diseases. 2009;68(1):8-17.

24. Meneses, SRF, Goode, AP, Nelson, AE, et al. Clinical algorithms to aid osteoarthritis guideline dissemination. Osteoarthritis and Cartilage. 2016;24(9):1487-1499.

25. Deveza LA, Melo L, Yamato TP, Mills K, Ravi V, Hunter DJ. Knee osteoarthritis phenotypes and their relevance for outcomes: A systematic review. Osteoarthritis and cartilage. 2017;25(12):1926-1941. 
26. Hochberg MC, Altman RD, April KT, et al. American college of rheumatology 2012 recommendations for the use of nonpharmacologic and pharmacologic therapies in osteoarthritis of the hand, hip, and knee. Arthritis care \& research (2010). 2012;64(4):465-474.

27. Jamison DE, Cohen SP. Radiofrequency techniques to treat chronic knee pain: A comprehensive review of anatomy, effectiveness, treatment parameters, and patient selection. Journal of pain research. 2018;11:18791888.

28. Bierma-Zeinstra S, Verhagen A. Osteoarthritis subpopulations and implications for clinical trial design. Arthritis research \& therapy. 2011;13(2):213.

29. Felson DT. Identifying different osteoarthritis phenotypes through epidemiology. Osteoarthritis and cartilage. 2010;18(5):601-604.

30. Centers for Medicare \& Medicaid Services. ICD-9-CM diagnosis and procedure codes: Abbreviated and full code titles. 2021. https://www.cms.gov/Medicare/Coding/ICD9ProviderDiagnosticCodes/codes. Accessed 2 December 2021.

31. Centers for Medicare \& Medicaid Services. ICD-10. 2021. https://www.cms.gov/Medicare/Coding/ICD10. Accessed 2 December 2021.

32. Ornetti P, Dougados M, Paternotte S, Logeart I, Gossec L. Validation of a numerical rating scale to assess functional impairment in hip and knee osteoarthritis: Comparison with the WOMAC function scale. Annals of the rheumatic diseases. 2011;70(5):740-746.

33. Illinois Bone \& Joint Institute (IBJI). Arthritis in knee: 4 stages of osteoarthritis. 2016. https://www.ibji.com/blog/orthopedic-care/arthritis-in-knee-4-stages-of-osteoarthritis/. Accessed 2 December 2021.

34. White DK, Neogi T, Nguyen UDT, Niu J, Zhang Y. Trajectories of functional decline in knee osteoarthritis: The osteoarthritis initiative. Rheumatology (Oxford, England). 2016;55(5):801-808.

35. National Institute for Health and Care Excellence, (NICE). Osteoarthritis overview. 2019. https://pathways.nice.org.uk/pathways/osteoarthritis. Accessed 2 December 2021.

36. Arthritis Foundation. Occupational therapy for arthritis. https://www.arthritis.org/healthwellness/treatment/complementary-therapies/physical-therapies/occupational-therapy-for-arthritis. Accessed 2 December 2021.

37. National Institute for Health and Care Excellence, (NICE). CG177: Osteoarthritis: Care and Management. 2014. https://www.nice.org.uk/guidance/cg177. Accessed 2 December 2021.

38. Bruyère $\mathrm{O}$, Honvo $\mathrm{G}$, Veronese $\mathrm{N}$, et al. An updated algorithm recommendation for the management of knee osteoarthritis from the european society for clinical and economic aspects of osteoporosis, osteoarthritis and musculoskeletal diseases (ESCEO). Seminars in arthritis and rheumatism. 2019;49(3):337-350.

39. Bruyère $\mathrm{O}, \mathrm{PhD}$, Cooper, Cyrus, MD, PhD, Pelletier J, MD, et al. A consensus statement on the european society for clinical and economic aspects of osteoporosis and osteoarthritis (ESCEO) algorithm for the management of knee osteoarthritis-From evidence-based medicine to the real-life setting. Seminars in arthritis and rheumatism. 2015;45(Suppl 4):3-11.

40. U.S. Bureau of Labor Statistics. Measuring price change in the CPI: Medical care. 2021. https://www.bls.gov/cpi/factsheets/medical-care.htm. Accessed 2 December 2021.

41. Van Spil WE, Bierma-Zeinstra SMA, Deveza LA, et al. A consensus-based framework for conducting and reporting osteoarthritis phenotype research. Arthritis research \& therapy. 2020;22(1):1-7. 
42. Bannuru RR, Osani MC, Vaysbrot EE, et al. OARSI guidelines for the non-surgical management of knee, hip, and polyarticular osteoarthritis. Osteoarthritis and cartilage. 2019;27(11):1578-1589.

43. Le TK, Montejano LB, Cao Z, Zhao Y, Ang D. Health care costs in US patients with and without a diagnosis of osteoarthritis. Journal of pain research. 2012;5:23-30.

44. Zhao X, Shah D, Gandhi K, et al. Clinical, humanistic, and economic burden of osteoarthritis among noninstitutionalized adults in the united states. Osteoarthritis and cartilage. 2019;27(11):1618-1626.

\section{Figures}

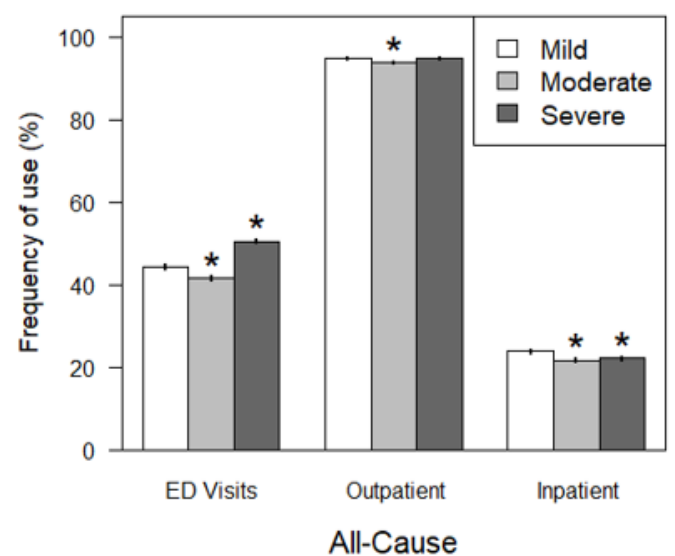

(a)

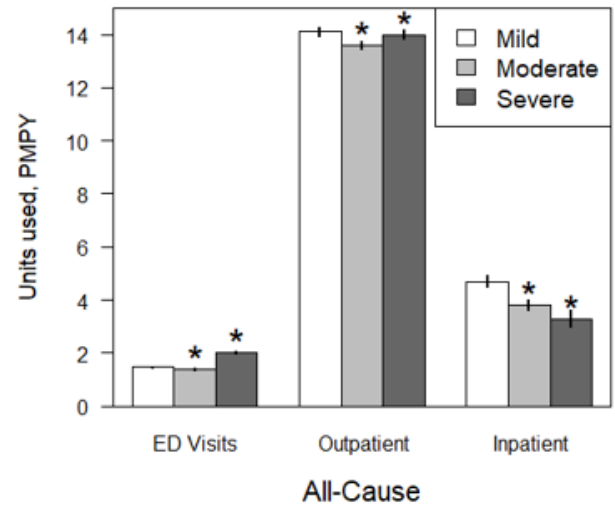

(c)

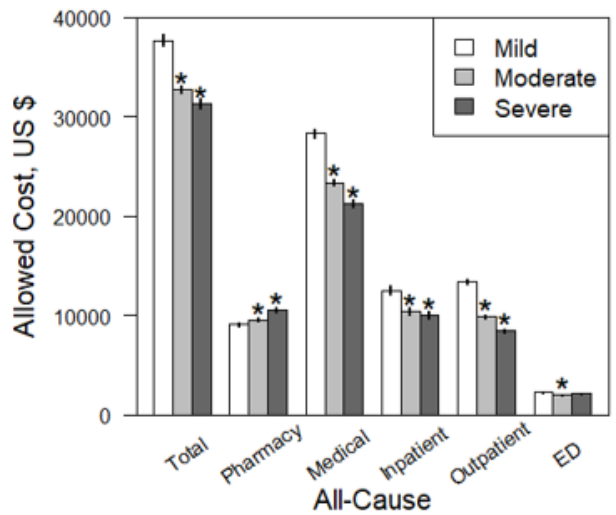

(e)

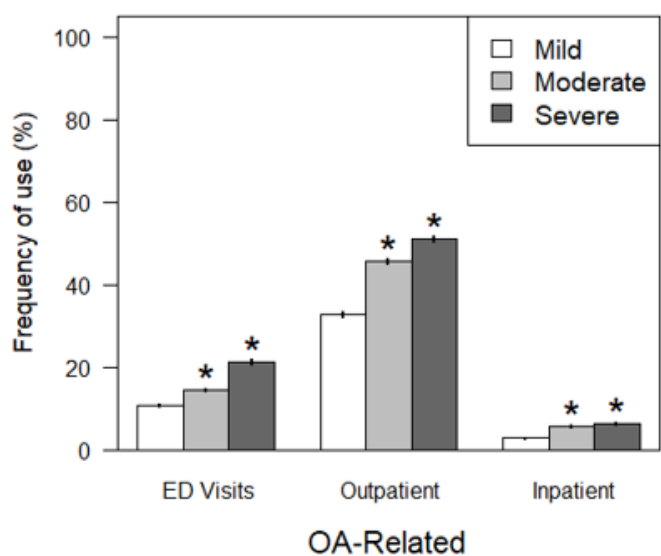

(b)

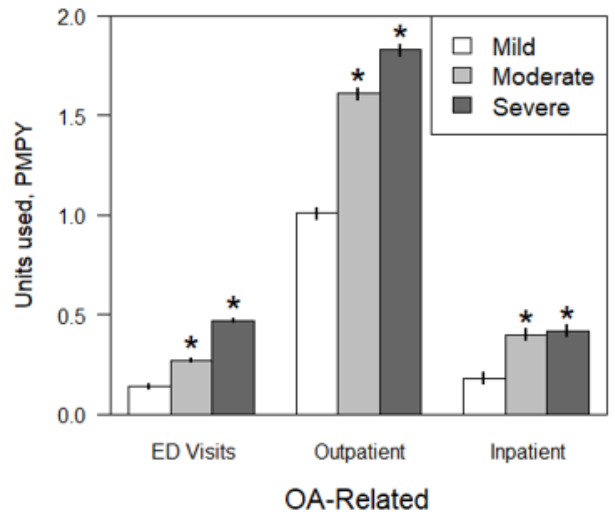

(d)

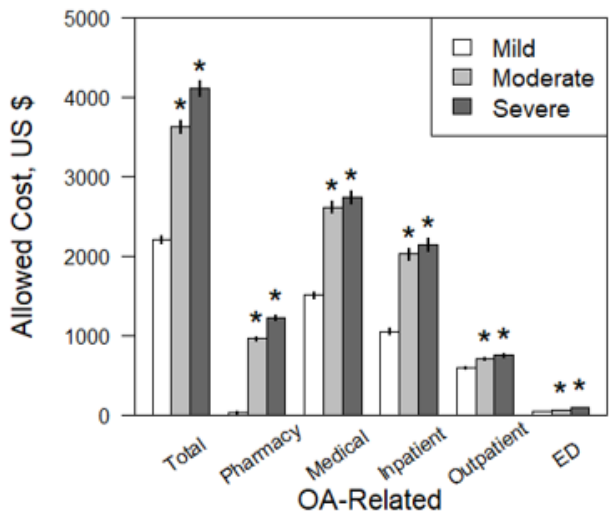

(f)

$\mathrm{ED}=$ emergency department visits, $\mathrm{OA}=$ osteoarthritis, $\mathrm{PMPY}=$ per member per year, US $=$ United States 
Utilization and cost outcomes, compared among three pain categories (mild, moderate and severe). Asterisks indicate significant contrasts (at $\mathrm{p}<0.0001$ ) between the indicated group compared to the mild pain category, and error bars indicate $95 \%$ confidence intervals. (a) Frequency (\% of patients with any use) of all-cause utilization. (b) Frequency of OA-related utilization. (c) Units used (PMPY rate), all-cause. (d) Units used (PMPY), OA-related. (e) Mean costs, all-cause. (f) Mean costs, OA-related.

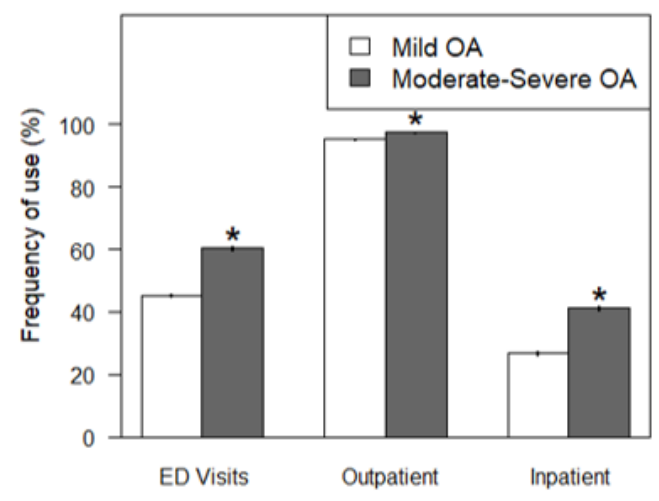

All-Cause

(a)

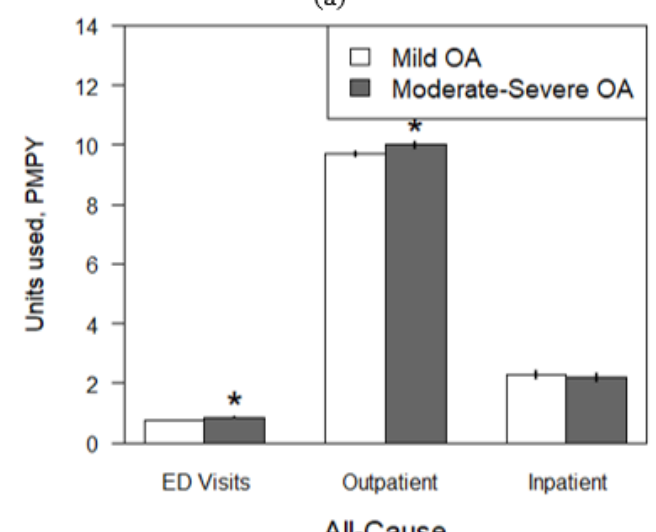

-Cause

(c)

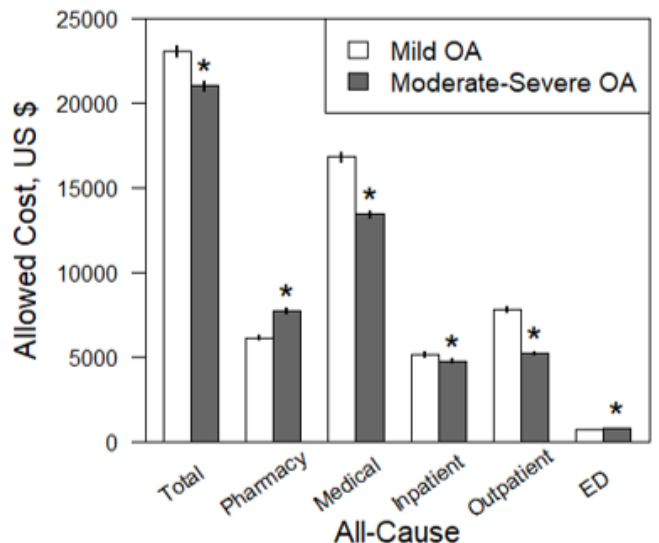

(e)

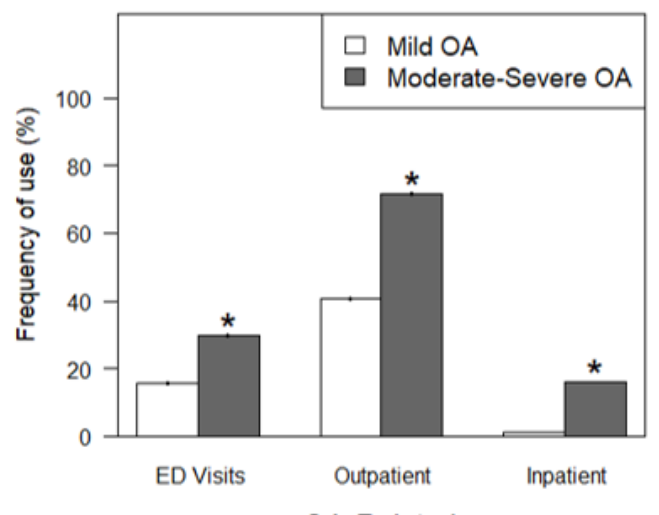

OA-Related

(b)

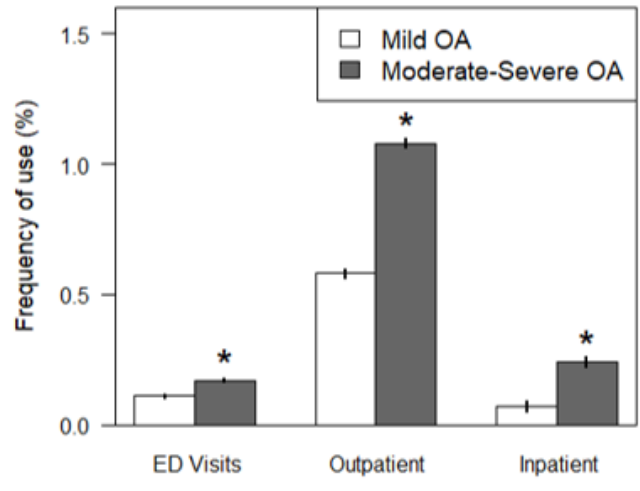

OA-Related

(d)

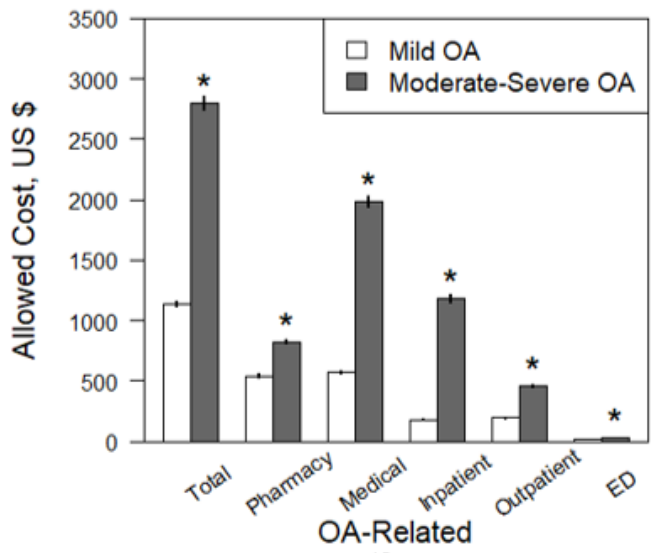

(f)

$\mathrm{ED}=$ emergency department visits, $\mathrm{OA}=$ osteoarthritis, $\mathrm{PMPY}=$ per member per year, US $=$ United States

\section{Figure 2}

Utilization and cost outcomes, compared among the two treatment-based categories (mild OA vs. moderate-severe $\mathrm{OA}$ ). Asterisks indicate significant contrasts compared to the mild OA category with $\mathrm{p}<0.0001$, and error bars indicate $95 \%$ confidence intervals. (a) Frequency (\% of patients with any use) of all-cause utilization. (b) Frequency 
of OA-related utilization. (c) Units used (PMPY rate), all-cause. (d) Units used (PMPY), OA-related. (e) Mean costs, allcause. (f) Mean costs, OA-related.

\section{Supplementary Files}

This is a list of supplementary files associated with this preprint. Click to download.

- APPENDIX.docx 